

Centro de Estudios de Arqueología Histórica

Universidad Nacional de Rosario

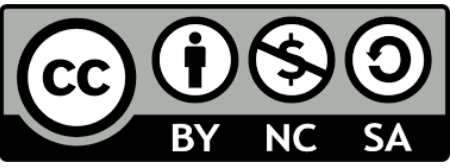

Teoría y Práctica de la Arqueología Histórica

Latinoamericana | Año X, Volumen 12 | 2021

Revista del Centro de Estudios de Arqueología Histórica, Facultad de Humanidades y Artes,

Universidad Nacional de Rosario

https://teoriaypracticaah.unr.edu.ar/index.php/index

https://rephip.unr.edu.ar/handle/2133/14804

ISSN en línea: 2591-2801

ISSN versión impresa: 2250-866X

Atribución-NoComercial-CompartirIgual 4.0 Internacional (CC BY-NC-SA 4.0)

https://creativecommons.org/licenses/by-nc-sa/4.0/deed.es

Alejandro García (ID: https://orcid.org/0000-0002-3537-

5879). Panorama de la Arqueología Histórica en Mendoza

\title{
Panorama de la Arqueología Histórica en Mendoza
}

\section{OVERVIEW OF HISTORICAL ARCHEOLOGY IN MENDOZA}

\author{
Alejandro García*
}

\section{Resumen}

La arqueología de tiempos históricos ha adquirido un marcado desarrollo en los últimos tiempos, y la provincia de Mendoza no ha estado al margen de este avance. Estos estudios comprenden tanto contextos indígenas como españoles-criollos y han sido efectuados por diversos investigadores y desde distintas perspectivas teóricas. Asimismo, abarcan distintos sectores geográficos de la provincia, sitios y actividades. A fin de tener una comprensión global de este desarrollo, en esta oportunidad se ofrece un breve panorama del estudio de sitios o contextos de época histórica a partir del análisis de algunos metadatos y se evalúan las claves del avance de la arqueología histórica mendocina registrado fundamentalmente en los últimos 30 años. Además se exploran algunos aspectos relacionados con la cantidad y calidad de la producción bibliográfica en este campo de estudio y con el papel e implicancias del posicionamiento teórico de los investigadores involucrados. El resultado del análisis constituye una descripción básica del estado de situación de estos estudios y ofrece un diagnóstico que permite detectar algunos puntos susceptibles de progreso.

Palabras clave: Arqueología histórica, Contextos históricos, Teoría arqueológica, Área fundacional, Mendoza

* Centro de Investigaciones de la Geósfera y Biósfera (Cigeobio). Conicet-Universidad Nacional de San Juan, Argentina. alegarcia@unsj.edu.ar 


\begin{abstract}
The archeology of historical times has acquired a marked development in recent times, and the province of Mendoza has not been left out of this advance. These studies cover both indigenous and Spanish-Creole contexts and have been carried out by various researchers and from different theoretical perspectives. They also cover various geographical sectors of the province and are linked to the analysis of very different sites and activities. In order to reach a global understanding of this development, in this occasion this paper offers a brief overview of the study of sites or contexts of historical times from the analysis of some metadata, and evaluate the keys to the advancement of the historical archeology of Mendoza recorded fundamentally in the last 30 years. In addition, it explores some aspects related to the quantity and quality of bibliographic production in this field of study, as well as the role and implications of the theoretical position of the researchers involved. The result of the analysis constitutes a basic description of the state of progress of these studies and offers a diagnosis that allows the detection of some points that can be improved.

Keywords: Historical archeology, Historical contexts, Archaeological theory, Foundational Area, Mendoza
\end{abstract}

\title{
Introducción
}

Desde mi perspectiva, hablar de Arqueología Histórica es lo mismo que referirse a Arqueología de tiempos o de momentos históricos (Goñi, 2000): simplemente se trata de una cuestión temporal, de una marca que indica la aparición de nuevos actores y de la generalización de los registros escritos (esta vez, en las lenguas de los nuevos protagonistas). De esta manera la mirada puede posarse sobre cualquier aspecto dentro un amplio abanico que abarca tanto el contacto hispano-indígena como la multiplicidad de situaciones vinculadas con el asentamiento, la subsistencia, la tecnología y las creencias de ambos bandos por separado, evitando los sesgos, prejuicios y explicaciones a priori relacionados con algunos enfoques recientes que enfatizan el papel de la expansión europea y del capitalismo (Deetz, 1996; Orser, 1996).

Los estudios arqueológicos de tiempos históricos han tenido un largo y variable desarrollo en la provincia de Mendoza. A fin de ofrecer una visión de conjunto sobre el tema, no enfocada exclusivamente en los contenidos mismos de los trabajos, en este artículo se bosqueja un panorama general de este desarrollo a partir del análisis de algunos metadatos de la producción bibliográfica correspondiente. Asimismo, se exploran algunos aspectos de interés relacionados con el avance cuanti-cualitativo de dicha producción y con la posición teórica de los investigadores que han explicitado sus enfoques, en vistas a la identificación de algunos elementos que permitan comprender mejor cómo se han realizado estos estudios.

\section{Breve crónica de las investigaciones}

Los primeros trabajos arqueológicos sobre contextos de tiempos históricos datan de la primera parte del siglo XX y corresponden a una serie de estudios realizados en el cementerio indígena de Viluco (Reed, 1919; Boman, 1920; Torres, 1923; Metraux, 1937; Rusconi, 1938). Algunas tumbas de este sitio brindaron elementos españoles (un cuchillo, un galón de oro, una medalla religiosa, etc.). Por esa época, Rusconi (1941) publicó algunos hallazgos de cerámica colonial y realizó una intensa tarea de recupe- 
ración de vestigios prehispánicos e históricos que hoy forman parte de las colecciones del Museo de Historia Natural "Juan Cornelio Moyano" de la ciudad de Mendoza. Años más tarde, las primeras excavaciones en un sitio no indígena de la provincia fueron practicadas en el Fuerte San Rafael del Diamante (1983, 2000, 2006) por Humberto Lagiglia, quien además sistematizó la información sobre la cerámica huarpe tardía (Viluco), cuya utilización se extendió a los primeros tiempos de la Colonia (Lagiglia, 1976).

En la década de 1990 hubo un desarrollo muy importante de la investigación de contextos de época histórica (Figura 1). Por un lado, Durán (1991-92, 1993-94, 1999, 2004) realizó estudios sobre los pehuenches del sur de Mendoza. Por el otro, diversos autores trabajaron en el Área Fundacional y en otros sitios del norte de Mendoza (e.g., Abal, 1993, 1998, 2002; Abal et al., 1996; Bárcena y Schávelzon, 1992; Bárcena, 1999, 2007; Schávelzon, 1998).

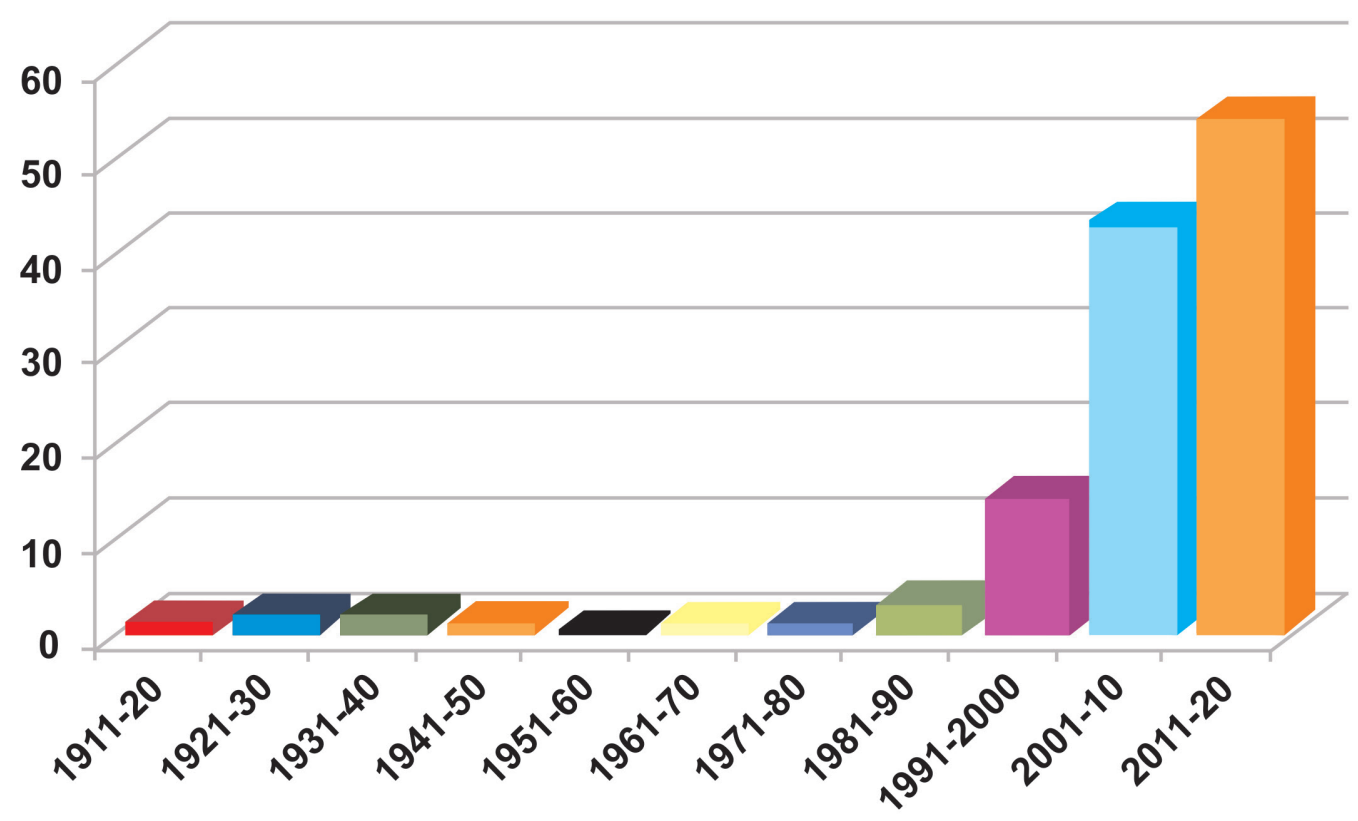

Figura 1. Cantidad de artículos de arqueología de tiempos históricos por década

En 1997 la Municipalidad de la Capital de Mendoza creó el Centro de Investigaciones de las Ruinas de San Francisco (CIRSF), institución que sería clave para el impulso de los estudios de Arqueología Histórica en la provincia durante las dos primeras décadas del siglo XXI. Las investigaciones de este centro se focalizaron en el Área Fundacional y alrededores (e.g., Chiavazza, 2005, 2011; Chiavazza y Zorrilla, 2005; Chiavazza et al., 2015). Como resultado se abordaron diversas temáticas específicas, entre las que sobresalen las condiciones de salud en tiempos históricos (e.g., Mansegosa, 2010, 2016, 2018; Giannotti, 2016, 2018) y la elaboración de la cerámica indígena y colonial en el área (e.g., Castillo, 2013; Prieto, 2010a, 2013; Puebla et al., 2005, 2008; Zorrilla y Puebla, 2010). Fuera del marco espacial de la ciudad, otros aspectos importantes analizados por los miembros del CIRSF son la configuración ambiental de la región a través de estudios arqueobotánicos (e.g. Mafferra, 2011, 2016, 2017) y la explotación minera colonial (e.g., Sironi 2010, 2015, 2019). Simultáneamente, otros investigadores han trabajado sobre estos y otros tópicos (e.g., Bárcena, 1999; Durán et al., 2003; García, 2011; García y Martínez Carre- 
tero, 2019; Gil et al. 2014; Novellino et al., 2003, etc.), aunque su actividad profesional no se encuentra estrecha y continuamente relacionada con los estudios de Arqueología Histórica.

\section{Análisis de algunas variables}

Una revisión detallada (aunque no exhaustiva) de la producción académica local referida a contextos de tiempos históricos (sin incluir textos divulgativos ni folletos) permitió conformar un conjunto de 127 entradas bibliográficas. La consideración de algunos datos permite tener un panorama general del tema.

Como se ha mencionado, los estudios de contextos de tiempos históricos abarcan todo el territorio provincial. Sin embargo, existen diferencias cuantitativas muy notables en las distintas regiones. Así, la región norte de Mendoza $\left(32^{\circ}-33^{\circ} 15^{\prime} \mathrm{S}\right)$ presenta una gran abundancia de estudios $(\mathrm{n}=104)$. En contrapartida, el centro $\left(33^{\circ} 15^{\prime}-35^{\circ} \mathrm{S} ; \mathrm{n}=17\right)$ y el sur $(n=6)$ han sido objeto de menos investigaciones. Esta diferencia no está dada sólo por una mayor cantidad de sitios investigados en el norte sino fundamentalmente por la realización de varios estudios sobre los mismos sitios y materiales arqueológicos provenientes del Área Fundacional y alrededores. Por ejemplo, al menos catorce artículos se refieren a trabajos específicos de cerámica indígena Viluco recuperada en esa zona. La incidencia de los estudios en dicha área se dimensiona fundamentalmente al considerar el sector étnico o social al que corresponden las muestras biológicas, materiales culturales o situaciones analizadas, ya que se observa un mayor peso cuantitativo

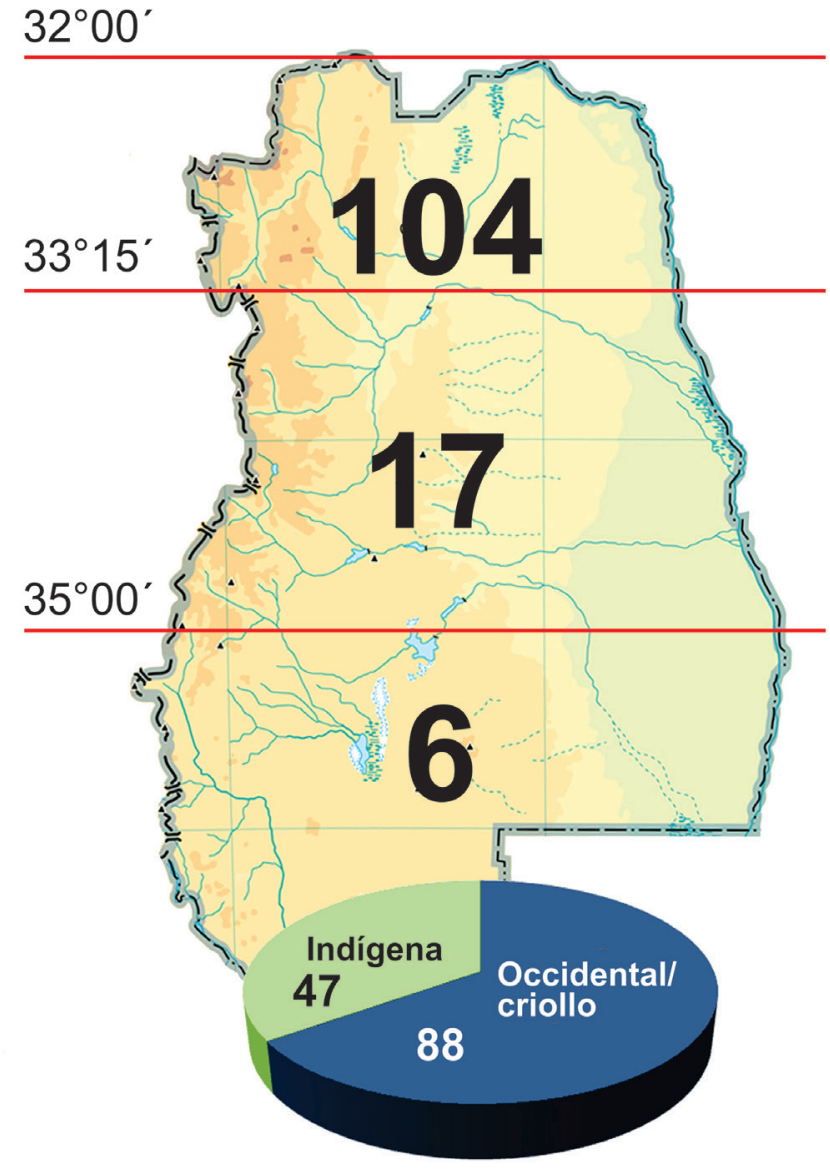
del grupo español-criollo residente en la ciudad (Figura 2). Además, el mayor peso cuantitativo de los estudios realizados en el sector norte coincide claramente con el momento de mayor producción de trabajos, esto es, durante las últimas dos décadas.

Figura 2. Producción bibliográfica sobre arqueología de tiempos históricos en Mendoza, según región y sector social analizado en los artículos 
En general los artículos relacionados con grupos indígenas involucran el estudio de cerámica Viluco recuperada en sitios nativos (Cápiz Alto y Viluco) y en sitios históricos urbanos, como las Ruinas de San Francisco, Alberdi e Ituzaingó y Edificio Plaza Huarpe. Por su parte, el predominio de publicaciones vinculadas con el sector occidental y criollo se basa en estudios referidos a aspectos bioarqueológicos, a la evolución del paisaje durante la Colonia y al tratamiento conjunto de los materiales provenientes de sitios mineros y urbanos. Dentro del espacio urbano, también se han realizado trabajos en la Iglesia y Convento de Santo Domingo y en varios predios cercanos (Bárcena, 2004), y en las iglesias de La Merced y San Agustín (Chiavazza, 2008). Esta situación se vincula con el hecho de que la mayor parte de los artículos se refiere precisamente al registro de sitios religiosos, ya sea de manera excluyente $(n=49)$ o en conjunto con otros tipos de localizaciones. Por su parte, la mayoría de las menciones de sitios considerados "administrativos" (Figura 3) corresponde al sector del Área Fundacional de la plaza y el Cabildo. Con respecto a los sitios indígenas, el que más trabajos reúne es el cementerio de Viluco, en San Carlos, con la particularidad de que esos artículos son en general individuales, más tempranos y corresponden a diversos autores (Reed, Boman, Torres, Metraux, Rusconi, Lagiglia), lo que contrasta con la pluralidad de autores en las publicaciones recientes.
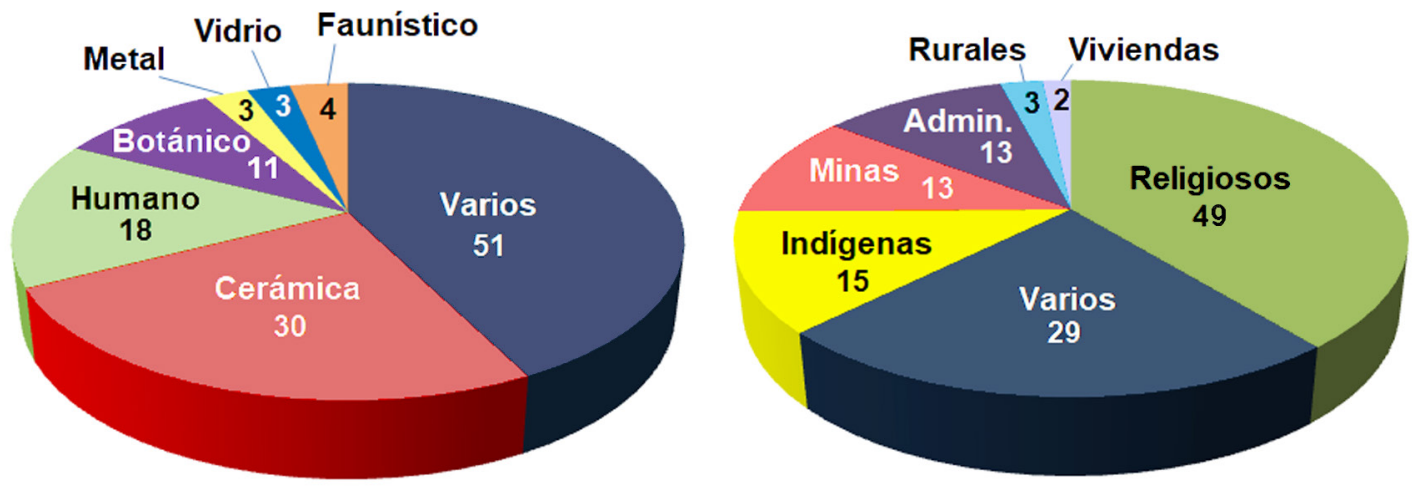

Figura 3. Cantidades de estudios arqueológicos de contextos coloniales según los materiales arqueológicos y clase de sitios analizados

Con respecto a la cronología, si bien en casi todos los artículos el abordaje de los contextos coloniales ocupa un lugar importante o excluyente, en algunos casos simplemente se trata de la integración de datos históricos a un marco temporal mucho mayor (Rusconi 1962; Gil et al. 2014; García y Martínez Carretero, 2019).

\section{Claves del avance de la Arqueología histórica en Mendoza}

Como ha podido observarse, las dos últimas décadas han atestiguado un avance significativo de las investigaciones vinculadas con contextos de época histórica en la provincia de Mendoza. Según uno de los principales protagonistas de esta nueva época, el hito fundamental del desarrollo de la Arqueología Histórica en el ámbito urbano de Mendoza fue la implementación de un proyecto, instancia que permitía 
superar las limitaciones de los trabajos anteriores. Estos antecedentes previos habrían producido información importante "pero el conocimiento generado tiende a ser parcelario, con temáticas analizadas desde disciplinas y fuentes que no han interactuado suficientemente dentro de una problemática común que propenda a explicar más que a describir el proceso" (Chiavazza, 2010a, p. 230). Así, los trabajos previos a la puesta en valor del Área Fundacional de Mendoza habrían perseguido un fin actualmente injustificado: "la detección de restos, (...) la descripción de secuencias y la adscripción de materiales a registros documentales" (Chiavazza y Tamiozzo, 2002, p. 133). Por el contrario, la nueva etapa de la arqueología urbana de Mendoza respondería a una noción de proyecto, cuyo propósito es "integrar la información arqueológica en un corpus que contribuya a la explicación del desarrollo urbano y de los modos de vida reflejados en los contextos materiales de producción, consumo y desecho de las pretéritas sociedades" (Chiavazza y Tamiozzo, 2002, p. 133). Este nuevo proyecto integra la Arqueología urbana con la Historia Ambiental, lo que genera expectativas vinculadas con la comprensión de "lo urbano como parte de un proceso que generó condiciones sanitarias, alimentarias y de interacción social en un punto del territorio que experimentó transformaciones" (Chiavazza, 2010a, p. 244).

En definitiva, según esta posición la clave para el avance significativo de la Arqueología Histórica mendocina, centrada en la arqueología urbana del Área Fundacional, habría sido la estructuración de los estudios dentro de un proyecto sólido. Sin embargo, desde una óptica más amplia, puede considerarse una serie de factores que brindan un panorama alternativo. En principio, cabe señalar que en general cualquier intervención arqueológica requiere la previa formulación y presentación de un proyecto, ya sea ante los organismos de financiación o ante las autoridades políticas locales. Por lo tanto la clave del progreso no puede ser la "noción de proyecto". En realidad, uno de los problemas principales de muchos proyectos durante el siglo XX y principios del siglo XXI fue el alcance de la financiación, no la ausencia de elaboración de planes con objetivos ambiciosos. La incidencia de este aspecto se refleja claramente en las advertencias de Durán et al. (2002, p. 199) acerca del alcance de los estudios realizados en el Paramillo de Uspallata:

Al encarar con recursos económicos escasos una problemática compleja (...) se tuvo en cuenta que era improbable poder alcanzar los objetivos más ambiciosos del proyecto en un corto plazo. Se dio prioridad entonces a algunas líneas de trabajo (prospección, sondeos exploratorios, armado de tipologías con fines cronológicos, etc.) destinadas a conformar un cuerpo de información de base que permitiera, en principio, discriminar y comparar los restos de los establecimientos del período estudiado de aquellos pertenecientes a momentos previos y posteriores (Durán et al. 2002, p. 199)

Sin duda, estas consideraciones son aplicables también a otros proyectos de duración y resultados limitados desarrollados en la región.

En relación con lo anterior, resulta evidente que una de las claves que explica el éxito de las investigaciones del CIRSF es el reconocido anclaje político-partidario de este centro, que garantiza una continuidad y estabilidad que lo diferencia de las demás iniciativas, cuya dependencia es exclusivamente académica. Desde esta óptica, el emprendimiento de referencia es más un programa político-cultural que un proyecto académico, lo cual se refleja ostensiblemente en la ausencia de objetivos específicos o problemas previos a resolver, los que han sido sustituidos por el objetivo general de conformar un "corpus que contribuya a la explicación del desarrollo urbano y de los modos de vida reflejados en los contextos materiales de producción, consumo y desecho de las pretéritas sociedades" (Chiavazza y Tamiozzo, 2002 , p. 133). Consecuentemente, en este caso parece claro que más que la formulación de un proyecto el factor fundamental fue el interés político de un municipio solvente, aunque también debe haber contribuido de manera decisiva la activa militancia dirigencial del director y fundador del centro (Chiavazza, 
actual Director de Patrimonio Cultural y Museos de la Provincia) dentro del mismo partido de cuyas filas han salido las autoridades encargadas del gobierno de la capital mendocina desde la década de 1980 (la Unión Cívica Radical).

Aun así, esto no alcanza para explicar la situación actual y la profunda transformación de la arqueología prehispánica e histórica mendocina de las últimas décadas, para lo cual es necesario considerar algunos factores críticos desarrollados a finales del siglo XX. Uno de ellos es la formación y disponibilidad de mayor cantidad de recursos humanos gracias a la creación de la Orientación Arqueología en la Licenciatura en Historia. Como resultado, durante las décadas de 1990 y 2000 surgieron en el norte de Mendoza decenas de nuevos arqueólogos ávidos de incursionar en el medio profesional, entre ellos el propio director y parte del grupo de trabajo del CIRSF. La diferencia cuantitativa con los cinco o seis arqueólogos que trabajaban en la Universidad Nacional de Cuyo en la década de 1980 (y que impulsaron dicha orientación) es clara.

Vinculado con lo anterior, no puede perderse de vista el importante aumento de los becarios graduados en la Universidad Nacional de Cuyo y de becarios doctorales locales de Conicet durante los últimos veinte años. Esta mayor oferta de posibilidades de financiación de estudios de posgrado fue acompañada por un marcado incremento de los subsidios de proyectos, lo que dio lugar a una base estructural totalmente novedosa para el avance de la disciplina a nivel regional.

Finalmente, este desarrollo de la investigación en el norte de Mendoza forma parte de un proceso evolutivo a escala nacional, caracterizado por la aparición de diversas especialidades dentro del marco de los estudios arqueológicos (análisis líticos, ceramológicos, bioarqueológicos, isotópicos, arqueobotánicos, de arte rupestre, etc.) que en poco tiempo configuraron un panorama diverso completamente diferente al que existía previamente.

En definitiva, el avance significativo de los estudios de arqueología de tiempos históricos en la provincia se asienta fundamentalmente en una serie de factores desarrollados desde la década de 1990, que brindaron dos elementos indispensables para la posterior aparición de un programa de estudios arqueológicos amparado por un organismo político importante: los recursos humanos especializados y el necesario grado de inserción de la arqueología en el medio. La actual situación, en consecuencia, es el resultado de la evolución de la disciplina en las últimas décadas, y en este marco no parece viable la comprensión del importante avance registrado en función de diferencias cualitativas con respecto a los proyectos de investigación previos sino más bien simplemente en relación a un aumento significativo de los estudios y de la producción académica.

\section{Cantidad no siempre implica calidad}

Es innegable que el aumento de producción bibliográfica sobre la arqueología de tiempos históricos representa un adelanto en el conocimiento local, y que la profundización del tratamiento de algunos temas refleja en sí misma un incremento de la calidad de la información disponible. Por ejemplo, esta situación se observa nítidamente en el caso de los estudios bioarqueológicos o en los estudios sobre cerámica de época colonial (tanto la indígena como la de origen occidental). De igual manera resultan evidentes las ventajas de contar con más trabajos relacionados con las reconstrucciones sobre paleovegetación y paleoambiente en el norte de Mendoza.

Sin embargo, es interesante observar la aparición reciente de algunos elementos que no son exclusivos de la Arqueología Histórica de Mendoza pero que de alguna manera forman parte del diagnóstico actual sobre el avance logrado y el alcance de los resultados obtenidos. Una de las razones más impor- 
tantes para hacer referencia a estos aspectos es que si bien no son muy recurrentes existe una tendencia generalizada a invisibilizarlos, aprovecharlos o ignorarlos. Entre los elementos más inocuos observados en la bibliografía sobre Arqueología Histórica local se destacan la reiteración en diversos trabajos de la misma información, a veces sin o con mínimos cambios gramaticales, y la forzada introducción de definiciones o aspectos teóricos que finalmente no guardan relación estrecha con los procedimientos analíticos específicos o con los resultados brindados por los artículos. Este último puede deberse a una necesidad de alineamiento ideológico institucional o a una firme convicción de que la presentación de tal información es imprescindible aunque no guarde relación estrecha con los trabajos y resultados a informar, algo probablemente heredado de la prédica de la Nueva Arqueología sobre la obligación de explicitar el posicionamiento teórico de los investigadores. La reiteración de contenidos, por su parte (junto con el fraccionamiento o atomización de la información), probablemente constituya una respuesta a los requerimientos de niveles de producción académica del mundo moderno, aunque es cierto que existen otras estrategias más adecuadas para cumplir con este tipo de exigencias.

Otros factores tienen implicancias académicas mayores, porque se relacionan más estrechamente con los resultados y las conclusiones. Entre ellos resaltan un alto grado de manejo de supuestos de investigación no corroborados, la reiteración de las mismas ideas o planteos en varios artículos de los mismos autores, la consideración sesgada o parcial de información previa y el bajo nivel de discusión externa de algunas propuestas. Algunos ejemplos son el uso de las incomprobables nociones de "equilibrio adaptativo" y "efectividad adaptativa" (ver infra), el pretendido paso de producción centralizada a descentralizada de la cerámica Viluco (Prieto, 2007, 2010a, b; Prieto y Chiavazza, 2009) y las ideas de que el área fundacional de Mendoza estaba habitada por los huarpes a la llegada del fundador de la ciudad y que la aculturación española de estos grupos se inició hacia 1540, o sea más de 20 años antes de la fundación de la ciudad (Chiavazza, 2008, 2010b).

En definitiva, ¿de qué dependen la validez, vigencia e importancia de los resultados? ¿Del renombre de las revistas en las que están publicados los trabajos? ¿De la cantidad de citas en trabajos de colegas? ¿Del idioma en que están escritos? ¿Del número de páginas y publicaciones que las expongan y reiteren? ¿De la falta de críticas o de propuestas alternativas? Aunque en la producción bibliográfica local a veces la presencia de estos elementos puede coincidir con un alto nivel de calidad de un trabajo, ninguno de ellos constituye la respuesta correcta: en general dependen de la calidad de los datos, de su criterioso manejo y de la fuerza del tejido de argumentaciones que los sustenta. En este sentido, lo que realmente garantiza el avance del conocimiento arqueológico sobre cualquier tema es la discusión abierta y desinteresada del proceso de obtención de los resultados y de las consecuentes interpretaciones, y, eventualmente, su confrontación con otras perspectivas o propuestas sobre el mismo tópico. Esta instancia de intercambio o discusión local tiene una importancia a veces subestimada, que no puede ser suplida automáticamente por otras prácticas. Por ejemplo, si bien la evaluación de artículos por parte de revisores nacionales o internacionales especializados aporta una especie de garantía en relación a aspectos metodológicos y a la vinculación a escala más amplia de los resultados, el manejo profundo de las problemáticas propias de un lugar muchas veces reside exclusivamente en los investigadores locales. Asimismo, el interés por analizar minuciosamente la solidez de la información de base en relación al conocimiento local a veces sólo se observa en los casos en que varios investigadores o grupos trabajan sobre una misma temática, lo que refleja la importancia de crear ámbitos de discusión efectivos previos a la instancia de publicación. Una vez publicado un trabajo sin haberse registrado tal discusión, deberían habilitarse vías que permitan la aparición de visiones alternativas o críticas. Las opciones existentes permiten a veces trascender las lógicas limitaciones impuestas por los conflictos de intereses personales o grupales, pero 
en definitiva dependen de las políticas editoriales de cada revista y en algunos casos de la discrecionalidad de los editores. Ejemplos positivos de este proceso y de un apropiado manejo editorial pueden verse en el intercambio de opiniones involucrado por algunos artículos referidos al sistema de subsistencia huarpe a la llegada de los españoles (Chiavazza y Mafferra, 2007; García, 2011) y en la divergencia de conclusiones acerca de los efectos del Último Máximo Glacial sobre la producción y consumo de maíz en Mendoza en tiempos coloniales (Gil et al., 2014; García y Martínez Carretero, 2019). Pero éstos son casos aislados de una práctica que debería ser mucho más frecuente y acompañar el armonioso crecimiento de la producción de información académica.

\section{El papel de la teoría}

Junto con los cambios antes señalados, al igual que lo observado a nivel nacional e internacional, en los últimos años se ha constatado una mayor diversificación de las posiciones teóricas de los arqueólogos mendocinos. Varios de los trabajos que nos atañen explicitan las preferencias o posicionamientos teóricos de los investigadores, aunque generalmente se trata de integrantes de un mismo grupo de trabajo. Un caso interesante es el de Bárcena (1995), quien a pesar de haber tratado específicamente el tema en un artículo sugerentemente denominado "De la Arqueología Histórica a la Arqueología como Arqueología" apenas brindó alguna definición sobre el tema. Este autor parece estar en desacuerdo con la diversificación de subdivisiones de la Arqueología, y no queda clara su posición frente a la Arqueología Histórica, aunque se muestra reticente a integrarla a la Historia y, en cambio, parece sugerir su afinidad con una "Arqueología antropológica, necesariamente orientada hacia lo social", que identifica con el materialismo histórico (Bárcena, 1995, p. 12,18). Llamativamente, esta posición no es desarrollada por el autor ni se ve reflejada en ninguno de sus trabajos arqueológicos, sean de época histórica o prehispánica.

Otra referencia sucinta fue realizada por Durán y colaboradores (2002, p. 202), para quienes la Arqueología Histórica es "una forma de hacer arqueología, que combina metodologías y técnicas propias con el análisis de documentación histórica". Más interesantes resultan dos acotaciones de estos investigadores: por un lado, su intento de encarar el estudio del sitio Minas de Paramillos desde una "perspectiva evolucionista próxima a las propuestas del procesualismo norteamericano" cuya aplicación habría encontrado algunos obstáculos (Durán et al., 2002, p. 199). Por el otro, los autores encuentran necesario explicitar sus diferencias con respecto a algunas "propuestas restrictivas" focalizadas en la dispersión europea en América, como las de Orser (1996) y Deetz (1996).

Por el contrario, éste es uno de los ejes principales del posicionamiento teórico de varios investigadores del CIRSF, para quienes la Arqueología Histórica se orienta a explicar la creación de un nuevo mundo a través de la dispersión de la cultura europea y de su impacto sobre las sociedades indígenas, de la imposición de un nuevo modo de vida, del comercio y de la Revolución Industrial (Romero et al., 2002; Chiavazza y Zorrilla, 2005; Puebla y Zorrilla, 2002). Este proceso habría provocado la ruptura del "equilibrio adaptativo" y de la "efectividad adaptativa" propuestas por Prieto (2000), que según Chiavazza et al. (2002, p. 93) se registran arqueológicamente para el período prehispánico final.

El otro eje teórico de este grupo está conformado por una adhesión a ciertos principios del materialismo histórico. Así, el concepto de Arqueología Histórica:

...debe avanzar en la consideración histórica y en su dimensión materialista y dialéctica. Tal abordaje requiere la definición de las contradicciones principales que giran en torno a los modos de organizar socialmente la producción y la reproducción. En este sentido, trabajo-naturaleza y hombres-mujeres son la base de cualquier ecuación (Chiavazza et al., 2002, p. 94). 
Estas contradicciones deben comprenderse mediante una dinámica dialéctica que determina la existencia de la vida: "cuando cesa la contradicción cesa la vida y se produce la muerte" (Chiavazza et al., 2002, p. 95).

En este marco ideológico:

...la base material está interdigitada por los demás aspectos sociales (creencias, ideologías) con los que interactúa según reglas de contradicción. De este modo no cabe el determinismo: la interacción es continua en tanto que dialéctica, desde el origen de los elementos constitutivos de la actividad social básica (Chiavazza, 2008, 229).

Por lo tanto, aun los valores, creencias y la vida espiritual son resultado de la interacción dialéctica de las condiciones materiales de vida. Diversas facetas de este mismo posicionamiento se reflejan en la producción de otros miembros del mismo centro de estudios (Puebla y Zorrilla, 2002; Romero et al., 2002; Sironi, 2014, Sironi et al., 2011, 2013; etc.)

Esta perspectiva parece enfrentar dos tipos principales de problemas. Con respecto al primer eje teórico, se observa un marcado sesgo constituido por la búsqueda de información comprobatoria de la expansión capitalista occidental a expensas de las sociedades indígenas locales. Como señala Goñi:

Las referencias a una causalidad última que se encuentra en la comprensión del sistema capitalista y su expansión, no es otra cosa que una interpretación esencialista y determinista de la arqueología, que le niega a la disciplina y al registro arqueológico la capacidad de dar explicaciones propias acerca del pasado (Goñi, 2000, p. 293).

No es que no sea válido plantear un estudio focalizado en la expansión capitalista como explicación del cambio, sino que esta visión resulta excluyente de todos los aspectos que no reflejen ese proceso, y dirige y condiciona a priori las interpretaciones hacia la verificación de los supuestos establecidos ideológicamente. Veamos un claro ejemplo. En un análisis arqueofaunístico de las Minas de Paramillos Sur, Sironi et al. (2013) presentan una cita de Haigh (1920, p. 56), quien señalaba que "para divertirnos y matar las horas convenimos en tomar alternativamente la escopeta y salir en busca de guanacos". Sin embargo, en las conclusiones la presencia de huesos de camélidos es interpretada negativamente como una acción probablemente debida a problemas de subsistencia ocasionados por "la no disponibilidad y acceso diferencial a alimentos permanentes" y la presencia de huesos de vaca, como prueba de un disciplinamiento capitalista de la dieta social (Sironi et al., 2013, p. 209-210). Esto significa que si los obreros comían carne vacuna estaban siendo disciplinados socialmente a través de la alimentación, y si comían carne de guanaco se debía a que se les limitaba la vacuna, o sea que en cualquier caso se destaca un elemento negativo (en una visión alternativa, se consideraría positivo que los obreros pudieran acceder a ambos tipos de carne). En el mismo sentido, el mantenimiento de algunos patrones decorativos en la cerámica Viluco de contextos hispánicos no es visto como parte de las estrategias positivas de adaptación nativa a las nuevas condiciones coloniales sino como fruto de incomprobables acciones de resistencia pasiva al poder español (Prieto y Chiavazza, 2009).

Por otra parte, la búsqueda de situaciones opuestas y conflictivas explicables a través de la dispersión del capitalismo occidental no siempre se realiza de una forma crítica que permita verificar los supuestos de base. Así, el conocimiento arqueológico de los tiempos prehispánicos tardíos es muy escaso y de ninguna manera indica un equilibrio adaptativo, especie de inocente y feliz línea de base nativa rousseauniana destrozada por la llegada de grupos occidentales. En realidad, la simple supervivencia 
de las sociedades indígenas locales es reflejo de su éxito o "efectividad adaptativa" a lo largo de miles de años y no una característica propia de los momentos inmediatamente prehispánicos. Por otro lado, la noción de equilibrio adaptativo deriva del supuesto de "una economía equilibrada entre la producción (agricultura y pastoralismo) y la extracción de recursos (pesca, caza y recolección)" (Prieto, 2000), una situación imposible de probar arqueológicamente para cualquier momento de la ocupación indígena de la región, ya que la simple aparición de evidencias relativamente sincrónicas de esas actividades no significa que hayan dado lugar a una economía armoniosa y equilibrada.

Con respecto al segundo eje, el principal problema es la propia cadena de supuestos sobre la que se apoya esta visión: así, a) la esencia de la vida y de la sociedad es el conflicto. b) El origen del conflicto es la diversidad (sexual, económica, social, etc.). c) El conflicto (y por lo tanto el desarrollo de la vida) ocurre siempre a través de pares opuestos, dicotómicos o dialécticos. d) Ese desarrollo puede ser íntegramente estudiado a través de los conflictos reflejados en variables dicotómicas. Evidentemente no todo en la vida es conflicto, y todas las instancias armoniosas del desarrollo social o cuyos agentes no sean pares dicotómicos quedan fuera de la órbita de análisis de esta perspectiva. Y aun en el caso de análisis de conflictos, no pueden abordarse aquellos que no presenten elementos contradictorios o dicotómicos. Por ejemplo, si los pares opuestos son hombre-mujer, empresario-obrero o español-indígena, quedan de lado los conflictos surgidos por la competencia entre empresarios o entre hombres, mujeres, nativos $\mathrm{u}$ obreros entre sí, a no ser que la contradicción alcance a todos los elementos sociales, que estarían en un permanente enfrentamiento interno.

Este inconveniente es asumido por uno de los investigadores del CIRSF, quien en un trabajo sobre arqueología industrial minera reconoce tener una concepción subjetiva y múltiple de la realidad, aunque acepta la aplicación de un recorte arbitrario que lo lleva a seleccionar sólo un par de factores: los medios de producción y los grupos domésticos involucrados en las actividades mineras (Sironi, 2014, p. 160). Este autor parecería querer trascender estas limitaciones, aunque denuncia su imposición por parte del sistema académico:

...utilizamos las herramientas analíticas y concebimos a la disciplina arqueológica según lo planteado por los autores [Palmer y Neaverson, 1998; Carandini, 1991 y Chiavazza y Zorrilla, 2005] sin haber reflexionado críticamente sus supuestos (teóricos, epistemológicos y ontológicos), ni ampliar la propuesta teórica-metodológica de los mismos. Es decir, que tomamos estos mensajes como absolutos, como recetas mágicas y únicas sin poder desprendernos de los rígidos sistemas preconcebidos por la lógica administrativa-financiera de los organismos científicos (Sironi, 2014, p. 161-162).

Este descontento con una aparente imposición de un determinado marco teórico para la realización de un proyecto específico parece reflejar una situación evidentemente anticientífica que puede tener implicancias negativas durante el análisis e interpretación final de los datos y, obviamente, afectar la calidad del proyecto.

\section{Consideraciones finales}

Esta breve revisión de algunos aspectos de la arqueología de momentos o tiempos históricos en Mendoza muestra que la misma ha tenido un avance importante en los últimos años, al que muchos arqueólogos locales han contribuido de diferentes formas. En un campo de estudio tan vasto, las posibilidades de aportar a la comprensión del pasado desde diversas ópticas son amplias. 
Desde el punto de vista teórico, mientras que para algunos autores (i.e., Lagiglia, Bárcena, Durán, García) el desarrollo de estudios de Arqueología Histórica simplemente resulta una aplicación de su perspectiva y metodología arqueológicas habituales a sitios más recientes, a veces con el aporte adicional de información documental (aunque Bárcena realice un planteo distinto), para los nucleados en el Centro de Investigaciones de las Ruinas de San Francisco parece implicar la necesidad de un posicionamiento ideológico manifiesto, vinculado con el materialismo histórico y la dispersión occidental/capitalista en América. Probablemente esta visión tendría una mejor articulación con la realidad si aplicara la tan reiterada y reverenciada dialéctica no sólo a la identificación de hipotéticos pares antinómicos en conflicto sino también a la discusión abierta de aspectos teóricos e interpretativos alternativos que permitieran trascender el encasillamiento ideológico de base.

Uno de los elementos cuya ausencia resalta en los estudios de sitios de tiempos históricos es la resolución de problemas específicos, esto es, el diseño de proyectos destinados directamente a dilucidar algún planteo puntual previo. Esta situación se observa claramente en relación a tópicos que involucran el trabajo nativo o el contacto hispano-indígena. Por ejemplo, las menciones documentales tempranas sobre la presencia de minas en Mendoza ameritan la búsqueda y estudio de indicadores de trabajo minero indígena prehispánico y colonial. Lo mismo sucede con la localización de las reducciones o pueblos de indios en el siglo XVIII, como el de Corocorto. Otro interesante problema a resolver arqueológicamente (más allá de la información documental) es si la ciudad de Mendoza se fundó o no en terrenos ocupados en ese momento por los indígenas, lo que habría ocasionado su traslado. En contrapartida, aun en el caso del programa de actividades del CIRSF, el enfoque metodológico es básicamente inductivo y se ciñe generalmente al análisis e interpretación de las evidencias que resulten de la excavación de las cada vez más escasas áreas excavables en el ámbito urbano.

Finalmente, aunque por varias razones es muy difícil replicar el caso del CIRSF, sería muy beneficiosa para la provincia la aparición y consolidación de otros grupos exclusiva o fundamentalmente dedicados a la Arqueología Histórica local. Esta situación probablemente traería aparejadas visiones diferentes sobre la disciplina y quizás la realización de nuevos trabajos en las áreas urbanas mendocinas, incluida la Capital. A su vez, estos elementos podrían incentivar la renovación y discusión de aspectos teóricos y metodológicos, promover el planteo de nuevos problemas y aportar interpretaciones alternativas que contribuyan desde la diversidad y heterogeneidad al mejoramiento de la comprensión del pasado histórico local.

\section{Agradecimientos}

Agradezco a Ana María Rocchietti y a la Comisión Organizadora del IX Simposio Nacional e Internacional Arqueología Histórica por su amable invitación a este encuentro. Extiendo mi gratitud a los evaluadores del trabajo, por sus constructivas consideraciones y sugerencias.

\section{Referencias bibliográficas}

Abal, C. (1993). Investigaciones arqueológicas en la Capilla de Nuestra Señora del Rosario -Rodeo de la Cruz, Guaymallén, Mendoza. Mendoza: Dirección de Patrimonio e Infraestructura Cultural. Municipalidad de Guaymallén.

Abal, C. (1998). Excavaciones Arqueológicas en San Francisco. Primera temporada. En D. Schávelzon, 
(Coord.), Las Ruinas de San Francisco (ex Jesuitas). Arqueología e Historia (pp. 67-102). Municipalidad de Mendoza, Mendoza.

Abal, C. (2002). La Capilla de Nuestra Señora del Rosario de Andacollo del Tulumaya, Lavalle, Mendoza. Investigación histórica y prospección. En Arqueología Histórica Argentina. Actas del $1^{\circ}$ Congreso Nacional de Arqueología Histórica (pp. 183-195). Mendoza: Corregidor.

Abal, C., Chiavazza, H., Contreras, O., Puebla, L. y Zorrilla, V. (1996). Arqueología Histórica Urbana, de Rescate, etc., etc., etc... La casa solariega de Don José A. Ozamis. Dpto. Maipú. Prov. Mendoza. Rca. Argentina. Historical Archaeology in Latin America, 16, 95-102.

Bárcena, J. (1995). De la arqueología histórica a la arqueología como arqueología. Comechingonia, 8, $5-20$.

Bárcena, J. (1999). Arqueología e Historia Urbana: investigaciones en el Área Fundacional de Mendoza, Departamentos Capital y Guaymallén. Actas del XII Congreso Nacional de Arqueología Argentina, tomo III (pp. 219-226). La Plata.

Bárcena, J. (2004). Arqueología e historia urbana: investigaciones en la ciudad y el conurbano mendocino. Chungara, 36 (supl. esp.), 187-196.

Bárcena, J. (2007). Arqueología e Historia: investigaciones en el predio de la iglesia y convento de Santo Domingo Soriano en Mendoza, Actas del XVI Congreso Nacional de Arqueología Argentina, tomo III, (pp. 287-294). San Salvador de Jujuy.

Bárcena, J. y Schávelzon, D. (1992). El Cabildo de Mendoza. Arqueología e Historia para su recuperación. Xama, 2, 1-174.

Boman, E. (1920). Cementerio indígena en Viluco (Mendoza) posterior a la conquista. Anales del Museo Nacional de Historia Natural de Buenos Aires, XXX, 501-559.

Carandini, A. (1991). Historias en la tierra: manual de excavación arqueológica. Barcelona: Crítica.

Castillo, L. (2013). Alfarería Indígena de Uso Doméstico en el Predio Ruinas de San Francisco del Área Fundacional de Mendoza. Mendoza: Editorial de la Facultad de Filosofía y Letras, Universidad Nacional de Cuyo.

Chiavazza, H. (2005). Arqueología en el predio mercedario de la ciudad de Mendoza. En H. Chiavazza y V. Zorrilla (Eds.), Arqueología en el predio mercedario de la ciudad de Mendoza (pp. 15-78). Mendoza: Facultad de Filosofía y Letras, Universidad Nacional de Cuyo.

Chiavazza, H. (2008). Bases teóricas para el análisis arqueológico de la espacialidad religiosa y los procesos de transformación cultural en la ciudad de Mendoza durante la colonia. Revista de Arqueología Americana, 25, 225-244.

Chiavazza, H. (2010a). Procesos sociales y ambientales en el sector urbano de Mendoza entre los siglos XV y XVIII: arqueología urbana e historia ambiental. Comechingonia virtual. Revista Electrónica de Arqueología, IV(2), 227-253.

Chiavazza, H. (2010b). Arqueología Histórica de la ciudad de Mendoza: explorando vínculos con Chile. 
Actas del XVII Congreso Nacional de Arqueología, Tomo 2 (pp. 1047-1058). Valdivia.

Chiavazza, H. (2011). El Área Fundacional de Mendoza. En M. Ramos, A. Tapia, F. Bognanni, M. Fernández, V. Helfer, C. Landa, M. Lanza, E. Montanari, E. Néspolo y V. Pineau (Eds.), Temas y problemas de la Arqueología Histórica. Luján: PROARHEP, Departamento de Ciencias Sociales, Universidad Nacional de Luján.

Chiavazza, H. y Tamiozzo, B. (2002). Arqueología a la vuelta de la esquina: excavaciones en la esquina de Alberdi e Ituzaingo. Arqueología Histórica Argentina. Actas del Primer Congreso Nacional de Arqueología Histórica Argentina (pp. 131-144). Buenos Aires: Corregidor.

Chiavazza, H., Puebla, L., Fiori, L., Ortega, C. y Hernández, F. (2002). Perspectiva arqueológica territorial: relaciones ciudad desierto desde los medanales de Lavalle. El caso de San José. Arqueología Histórica Argentina. Actas del Primer Congreso de Arqueología Histórica Argentina (pp. 89-111). Buenos Aires: Corregidor.

Chiavazza, H. y Zorrilla, V. (2005). Arqueología en el predio mercedario de la ciudad de Mendoza. Mendoza: Editorial Facultad de Filosofía y Letras, Universidad Nacional de Cuyo.

Chiavazza, H. y Maferra, L. (2007). Estado de las investigaciones arqueobotánicas en Mendoza y sus implicancias en la arqueología histórica. Revista de Arqueología Histórica Argentina y Latinoamericana, 1, 127-152.

Chiavazza, H., Mansegosa, D., Gámez Mendoza, A. y Giannotti, S. (2015). Funebria católica y estimaciones del sexo y de la edad en entierros de una ciudad americana colonial (Mendoza, Argentina, siglos XVII-XIX). Revista de Arqueología Histórica Argentina y Latinoamericana, 9(1), 35-70.

Deetz, J. (1996). In Small Things Forgotten: An Archaeology of Early American Life. New York: Anchor Books.

Durán, V. (1991-1992). Las poblaciones indígenas del sur mendocino durante los siglos XVI y XVII. Anales de Arqueología y Etnología, 46/47, 9-40.

Durán, V. (1993-1994). La araucanización de las poblaciones indígenas del sur mendocino (siglos XVIII y XIX). Anales de Arqueología y Etnología, 48/49, 31-55.

Durán, V. (1999). Los Pehuenches Malargüinos. Una aproximación histórica y su contrastación arqueológica. Revista de Estudios Regionales, 19, 119-161.

Durán, V. (2004). Poblaciones indigenas de Malargüe. Su arqueología e historia. Mendoza: Centro Interdisciplinario de Estudios Regionales, Universidad Nacional de Cuyo.

Durán, V., Altamira, M., Vega, B., Zarandón, N., Rey, A. y Ulloa, P. (2002). Eran unas minas de plata. Arqueología e historia de la minería del siglo XIX en la precordillera mendocina. Arqueología Histórica Argentina. Actas del Primer Congreso Nacional de Arqueología Histórica (pp. 199-216). Mendoza: Corregidor.

Durán, V., Figueroa, P., Gasco, A., Altamira, M., Rey, A., Estrella, D., Vega, B., Zarandón, N. y Pantanetti, M. (2003). Análisis arqueológico de la minería industrial de fines del siglo XIX en el Paramillo 
de Uspallata. En A. Cueto (Comp.), Minería e impacto en Mendoza (pp. 153-203). Mendoza: CEIDER, Facultad de Filosofía y Letras, Universidad Nacional de Cuyo.

García, A. (2011). Agricultura huarpe y conquista española: discusión de recientes propuestas. Revista de Arqueología Histórica Argentina y Latinoamericana 5:147-163.

García, A. y Martínez Carretero, E. (2019). Corn consumption in native populations of Mendoza (centralwestern Argentina) and its relation to environmental conditions. Multequina, 28, 5-20.

Giannotti P.S. (2016). Marcadores de estrés ocupacional en poblaciones históricas del norte de Mendoza (s. XVI-XVII): primeros resultados exploratorios. Comechingonia, 20(1), 81-110.

Giannotti P.S. (2018). Aproximación a las condiciones de salud en la sociedad estamental mendocina a partir del análisis bioarqueológico: Templo La Caridad (s. XVIII-XIX). [Dossier] Revista de Arqueología Histórica Argentina y Latinoamericana, 12, 559-588.

Gil, A., Villalba, R., Ugan, A., Cortegoso, V., Neme, G., Michieli, C., Novelino P. y Durán, V. (2014). Isotopic evidence on human bone for declining maize consumption during the Little Ice Age in central western Argentina. Journal of Archaeological Science, 49, 213-227.

Goñi, R. (2000). Arqueología de Momentos Históricos fuera de los centros de conquista y colonización: un análisis de caso en el sur de Patagonia. En Desde el país de los gigantes. Perspectivas arqueológicas en Patagonia, tomo 1 (pp. 283-296). Río Gallegos: Universidad Nacional de la Patagonia Austral.

Haigh, S. (1920). Bosquejos de Buenos Aires, Chile y Perú. Buenos Aires: La Cultura Argentina.

Lagiglia, H. (1976). La Cultura de Viluco del Centro Oeste Argentino. Revista del Museo de Historia Natural, III(1/4), 227-265.

Lagiglia, H. (1983). Presencia hispánica en la minería indígena y colonial de Mendoza. En Presencia Hispánica en la Arqueología Argentina t.1, pp. 205-227. Resistencia: Universidad Nacional del Nordeste.

Lagiglia, H. (2000). Arqueología histórica: la guimbarda en la línea de frontera. Notas del Museo, 44, $1-34$.

Lagiglia, H. (2006). Arqueología e Historia del Fuerte San Rafael del Diamante (Mendoza). San Rafael: Museo de Historia Natural.

Mafferra, L. (2011). Interpretaciones del registro arqueobotánico en arqueología histórica. En M. Ramos, A. Tapia, F. Bognanni, M. Fernández, V. Helfer, C. Landa, M. Lanza, E. Montanari, E. Néspolo y V. Pineau, (Eds.), Temas y problemas de la Arqueología Histórica, Tomo 2 (pp. 43-52). Luján: PROARHEP, Departamento de Ciencias Sociales, Universidad Nacional de Luján.

Mafferra, L. (2016). Arqueobotánica del Norte de Mendoza. Interpretaciones sobre el rol de los vegetales en la interacción indigena-hispana durante los siglos XVI y XVII. Mendoza: Facultad de Filosofía y Letras de la Universidad Nacional de Cuyo.

Mafferra, L. (2017). Los paisajes forestales en torno a la ciudad colonial de Mendoza, con base en el registro antracológico. Intersecciones en Antropología, XVIII(1), 43-53. 
Mansegosa, D. (2010). Estudios bioarqueológicos en un templo colonial de Mendoza: La Caridad. XVII Congreso Nacional de Arqueología Argentina, Tomo 5 (pp. 1777-1782). Mendoza: Facultad de Filosofía y Letras, Universidad Nacional Cuyo e INCIHUSA.

Mansegosa, D. (2016). Estudios sobre salud y enfermedad en poblaciones históricas urbanas de Mendoza (Argentina). Nuevos aportes para el estudio bioarqueológico del templo La Caridad. Comechingonia, 20 (1), 111-142.

Mansegosa, D. (2018). Dieta y condiciones de salud oral en poblaciones históricas del norte de Mendoza. [Dossier] Revista de Arqueología Histórica Argentina y Latinoamericana, 12, 589-615.

Metraux, A. (1937) [1929]. Contribución a la etnografía y arqueología de la provincia de Mendoza. Revista de la Junta de Estudios Históricos de Mendoza, VI (15-16), 1-66.

Novellino, P., Durán, V. y Prieto, C. 2003. Cápiz Alto: aspectos bioarqueológicos y arqueológicos del cementerio indígena de época postcontacto (provincia de Mendoza, Argentina). Paleopatología, 1, $1-16$.

Orser, Ch. (1996). A historical archaeology of the modern world. New York: Plenum Press.

Palmer, M. y Neaverson, P. 1998. The scope of industrial archaeology. En M. Palmer y P. Neaverson (Eds.), Industrial Archaeology Principles and Practice (pp. 1-15). Londres: Routledge.

Prieto, M. del R. (2000). Formación y consolidación de una sociedad en un área marginal del Reino de Chile: la Provincia de Cuyo en el siglo XVII. Anales del Instituto de Arqueología y Etnología, $52-$ $53,18-366$.

Prieto Olavarría, C. (2007). Cambios en la producción cerámica Viluco en los siglos XV y XVII. Norte y centro de Mendoza. Actas del XVI Congreso Nacional de Arqueología Argentina, tomo II (pp. 389-395). Jujuy

Prieto Olavarría, C. (2010a). La cerámica Viluco en el norte y centro de Mendoza: producción e interacciones. Actas del XVII Congreso Nacional de Arqueología Chilena, Tomo I (pp. 403-410). Valdivia.

Prieto Olavarría, C. (2010b). Aproximaciones a la producción y función de la cerámica Viluco durante la dominación incaica y los primeros siglos de la colonia en el valle de Mendoza. En Actas del XVII Congreso Nacional de Arqueología Argentina (pp. 205-210). Mendoza: Facultad de Filosofía y Letras, Universidad Nacional Cuyo e INCIHUSA.

Prieto Olavarría, C. (2013). La presencia indígena en la ciudad de Mendoza en los siglos XVI y XVII. Análisis desde la evidencia cerámica y etnohistórica. Actas del V Congreso Nacional de Arqueología Histórica, tomo 2 (pp. 9-39). Saarbrücken: Editorial Académica Española.

Prieto Olavarría, C. y Chiavazza, H. (2009). La producción cerámica Viluco entre los siglos XV y XVII (Provincia de Mendoza, Argentina). Chungara, 41(2), 261-274.

Puebla, L. y Zorrilla, V. (2002). Aproximaciones a la variabilidad de productos cerámicos de manufactura occidental procedentes del Área Fundacional de la ciudad de Mendoza. Arqueología Histórica 
Argentina. Actas del Primer Congreso Nacional de Arqueología Histórica (pp. 163-173). Mendoza: Corregidor.

Puebla, L., Zorrilla, V. y Chiavazza, H. (2005). Análisis del material cerámico histórico del predio mercedario de la ciudad de Mendoza. En H. Chiavazza y V. Zorrilla (Eds.), Arqueología en el predio mercedario de la ciudad de Mendoza (pp. 157-218). Mendoza: Facultad de Filosofía y Letras, Universidad Nacional de Cuyo.

Puebla, L., Zorrilla, V. y Chiavazza, H. (2008). Mendoza en el periodo Colonial Temprano: Mayólicas y Cerámicas locales. Actas del tercer Congreso Nacional de arqueología Histórica (pp. 658-666). Rosario: Universidad Nacional de Rosario.

Reed, C. (1919). Cementerio indígena postcolombino de Viluco, provincia de Mendoza. Physis, IV, 9496.

Romero, A., Hernández, F. y Barboza, D. (2002). Arqueofauna; enfoques y estudios en el espacio fundacional de Mendoza. Arqueología Histórica Argentina. Actas del Primer Congreso de Arqueología Histórica Argentina (pp. 153-161). Corregidor: Buenos Aires.

Rusconi, C. (1938). El material arqueológico de Viluco es en su totalidad de época post-hispánica? Anales del Primer Congreso de Historia de Cuyo, tomo IV (pp. 439-445). Mendoza.

Rusconi, C. (1941). Alfarería colonial de Mendoza. Actas del Congreso de Historia Argentina Norte y Centro, tomo 1 (pp. 257-267). Córdoba: Academia Nacional de Historia Filial de Córdoba.

Rusconi, C. (1962). Poblaciones pre y post hispánicas de Mendoza. Volumen III "Arqueología". Mendoza: Edición Oficial.

Schávelzon, D. (coord.) (1998). Las Ruinas de San Francisco. Arqueología e Historia. Mendoza: Municipalidad de Mendoza.

Sironi, O., Chiavazza, H. y García, V. (2011). El registro vítreo del matadero público de Mendoza (18771927). Revista de Arqueología Histórica Argentina y Latinoamericana, 5, 95-124.

Sironi, O., Araujo, E., López, M. y Quiroga, M. (2013). Arqueozoología de un contexto minero: Minas Paramillos Sur (Mendoza, Argentina). Comechingonia Virtual, VII(2), 189-215.

Sironi, O. (2010). Los diversos usos del vidrio en un emplazamiento minero de Precordillera (Provincia de Mendoza). Cuadernos de Antropología, 5, 189-220.

Sironi, O. (2014). Arqueología histórica industrial: Propuesta epistemológica y metodológica para una arqueología de la minería. Entelequia Revista Interdisciplinar, 17, 155-168.

Sironi, O. (2015). Intervenciones arqueológicas en el sitio Minas Paramillos Sur (Reserva Natural Villavicencio, Mendoza). En J.R. Bárcena (Ed.), Arqueología y Etnohistoria del Centro Oeste Argentino: aportes desde las V Jornadas Arqueológicas Cuyanas (pp. 119-133). Mendoza.

Sironi, O. (2019). Mining Ways of Life in the Southern Andes: Historical Anthropological Archaeology in Mendoza, Argentina". International Journal of Historical Archaeology, 23(1), 153-171. 
Torres, L. (1923). Exploraciones arqueológicas al sur de San Carlos (provincia de Mendoza). Revista del Museo de La Plata, XXVII, 286-305.

Zorrilla, V. y Puebla, L. (2010). Aportes metodológicos para el estudio de cerámicas coloniales en Mendoza. Arqueología Argentina en el Bicentenario de la Revolución de Mayo. Actas del XVII Congreso Nacional de Arqueología Argentina, Tomo I (pp. 211-215). Mendoza: Facultad de Filosofía y Letras, Universidad Nacional Cuyo e INCIHUSA.

Recibido 4 de Diciembre 2020

Aceptado: 30 de Abril 2021 